\title{
Smooth Haired Mini Dachshund
}

National Cancer Institute

\section{Source}

National Cancer Institute. Smooth Haired Mini Dachshund. NCI Thesaurus. Code C53902.

The Smooth Haired Mini Dachshund is a smooth-haired variety of dachshund with a height up 5 inches. 